\title{
Optimization and characterization of femtosecond laser inscribed in- fiber microchannels for liquid sensing
}

\author{
Graham C. B. Lee*, Chengbo Mou, Kaiming Zhou and Kate Sugden \\ Aston Institute of Photonic Technologies, Aston University, Birmingham, UK
}

\begin{abstract}
In-fiber microchannels were fabricated directly in standard single mode fiber using the femtosecond laser inscribe and etch technique. This method of creating in-fiber microchannels offers great versatility since it allows complex three dimensional structures to be inscribed and then etched with hydrofluoric acid. Four in-fiber microchannel designs were experimentally investigated using this technique. Device characteristics were evaluated through monitoring the spectral change while inserting index matching oils into each microchannel - a R.I. sensitivity up to $1.55 \mathrm{~dB} / \mathrm{RIU}$ was achieved. Furthermore, a simple Fabry-Pérot based refractometer with a R.I. sensitivity of $2.75 \mathrm{~nm} / \mathrm{RIU}$ was also demonstrated.
\end{abstract}

Keywords: microchannel, fiber optic sensors, refractometer, femtosecond laser, inscription, etching, hydrofluoric acid.

\section{INTRODUCTION}

In-fiber microchannels exploits the diverse sensing capability of optical fibers to interrogate liquids. Optical fibers are well suited for sensing in harsh environments, which make them well positioned for sensing hazardous liquids. Similarly, the size and flexibility of optical fibers allow them to be installed in most locations. Optical fibers have previously been used to enable liquid and gas sensing. Jensen et al. were able to interrogate liquids using photonic-crystal fiber (PCF) by injecting a fluid into the cladding holes [1]. Likewise, Lien and Vollmer demonstrated an integrated microfluidic flow sensor based on a fiber-tip cantilever [2]. While Perrotton et al. built a sensitive optical fiber hydrogen sensor based on surface plasmon resonance [3]. The recent development of femtosecond lasers have allowed novel structures and designs to be written in optical fibers to create new fiber optic sensors. Femtosecond lasers can create highly accurate and highly localized permanent changes in both opaque and dielectric transparent materials through several non-linear processes [4]. The interaction between a femtosecond laser pulse and a dielectric transparent material can result in a change in refractive index (R.I), birefringence or the creation of a void. The type of modification is chosen through the careful control of a vast number of parameters, such as the laser pulse duration, laser fluence and the focusing condition for a given material. In-fiber microchannels fabricated using the femtosecond laser inscribe and etch technique was first demonstrated by Lai et al. in 2006 [5]. Lai and co-workers inscribed and preferentially etched a single $4 \mu \mathrm{m}$ wide microhole through the fiber core and cladding. In this paper, the work of Lai et al. [5] was extended to explore and characterize more complex microchannel designs. Four in-fiber microchannel designs were experimentally investigated in standard single mode fiber using the inscribe and etch technique. In addition, a simple Fabry-Pérot (FP) based refractometer was demonstrated through the inscription of two identical point-by-point fiber Bragg gratings (FBGs) [6] with a microchannel situated in the intrinsic cavity.

\subsection{Femtosecond laser and setup}

The microchannel and refractometer devices were inscribed using an Amplitude Systèmes s-Pulse HP femtosecond laser that produces sub $500 \mathrm{fs}$ laser pulses at a center wavelength of $1026 \mathrm{~nm}$. The microchannel devices and the FBGs were inscribed using a repetition rate of $100 \mathrm{kHz}$ and $1 \mathrm{kHz}$ respectively. All inscriptions were conducted using a $100 \mathrm{x}$ objective (Mititoyo MPlan Apo NIR Series) with a numerical aperture of 0.5. The high NA lens offered a long working distance, which generates a small and highly accurate focal spot.

*leegcb@aston.ac.uk; phone 44121204 3545; www.aston.ac.uk

23rd International Conference on Optical Fibre Sensors, edited by José Miguel López-Higuera,

Julian Jones, Manuel López-Amo, José Luis Santos, Proc. of SPIE Vol. 9157, 915743

(C) 2014 SPIE · CCC code: $0277-786 X / 14 / \$ 18 \cdot$ doi: $10.1117 / 12.2058315$ 
The approximate FWHM of the laser spot size incident on the samples was approximately $1.5 \mu \mathrm{m}$. All devices were fabricated in standard single mode fiber (Corning SMF-28e). Fiber samples were secured to a standard microscope slide with a small section of the acrylate coating removed to facilitate the inscription. A small quantity of index matching oil was applied to the area of the fiber where the coating was removed and a cover slip was placed on top of the oil. This fiber mounting configuration was used to reduce the defocusing-induced distortions created by the curvature of the fiber to allow the femtosecond laser beam to remain tightly focused during inscription. The fiber samples secured to the microscope slide were mounted to an Aerotech motion control system (X-Y Aerotech ABL1000 air bearing stages and mechanical Z Aerotech ANT-4V stage) that was controlled with a custom written CNC program.

\section{DESIGN}

With reference to Figure 1, four in-fiber microchannels designs were implemented using the inscribe and etch technique. In addition, Figure 2 shows the design of a simple FP based refractometer formed by two point-by-point FBGs separated by a given length with a microhole positioned between the two gratings.

(a)

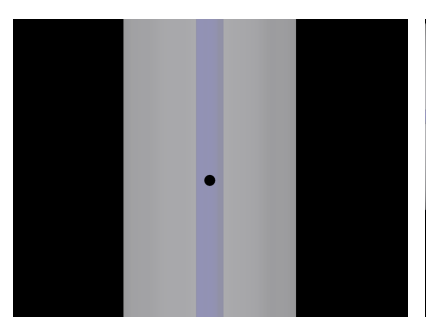

(b)

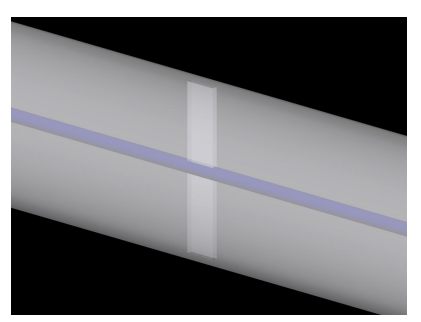

(c)

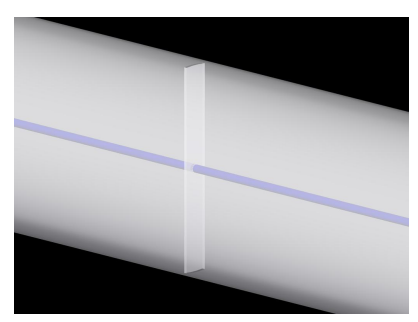

(d)

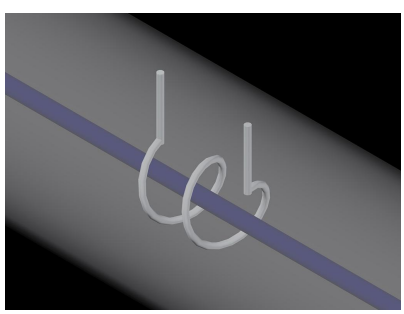

Figure 1. CAD render of the in-fiber microchannel designs: (a) microhole, (b) microslot channel along the core, (c) microslot channel perpendicular to the core and (d) single spiral channel around the core.

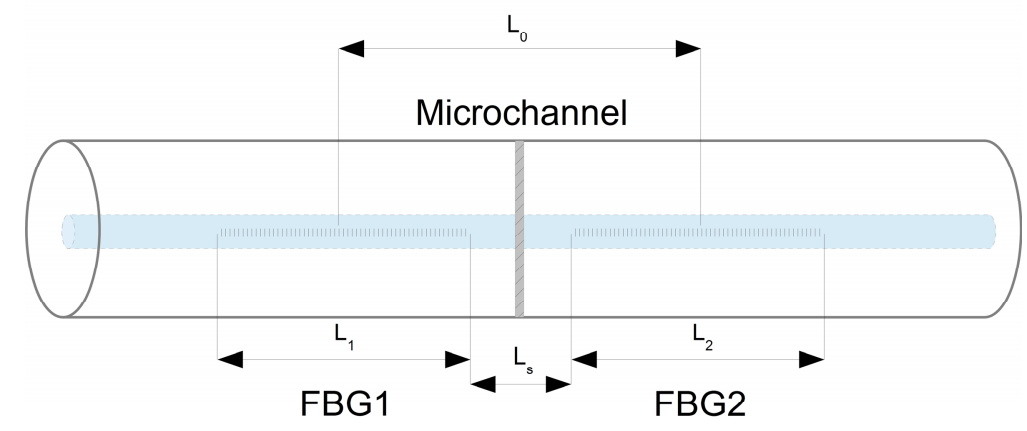

Figure 2. FP resonator in standard SMF formed by two FBGs (FBG1 and FBG2), $\mathrm{L}_{1}$ and $\mathrm{L}_{2}$ are the respective lengths of the gratings. $L_{0}$ is the cavity length (center to center length of the two gratings). $L_{s}$ is the separation between the last grating plane of FBG1 and first grating plane of FBG2

The microhole design was inscribed by translating the laser beam from the bottom of the fiber, through the core and to the top of the fiber in one smooth motion. The microslot channels were fabricated through inscribing the desired length microslot in the XY plane and then writing the same microslot layer by layer in the $\mathrm{Z}$ plane from the bottom to the top of the fiber. The spiral channel around the core was formed by translating the laser beam around the core while maintaining a given distance from the center of the core; microholes were also fabricated at the two ends of the spiral to allow liquids to enter the microchannel. 


\section{RESULTS AND DISCUSSION}

The dimensions of the inscribed microchannel can be tailored by adjusting the number of laser passes, pulse energy delivered and inscription speed for a given lens and repetition rate. The microhole, microslots and helix designs were inscribed with a pulse energy of $120 \mathrm{~nJ}-180 \mathrm{~nJ}$ at a translation speed of $0.2 \mathrm{~mm} / \mathrm{s}$. After femtosecond laser inscription, each microchannel device was etched in a $5 \%$ hydrofluoric acid solution assisted by an ultrasonic bath, the approximate etch time required for each device were: microhole (25 minutes), microslots (10 to 60 minutes) and helix (60 minutes). The fully etched microchannel devices are shown in the microscope images presented in Figure 3.

(a)

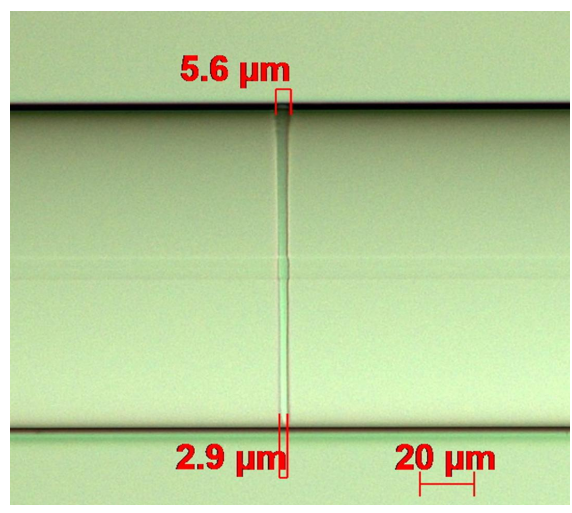

(c)

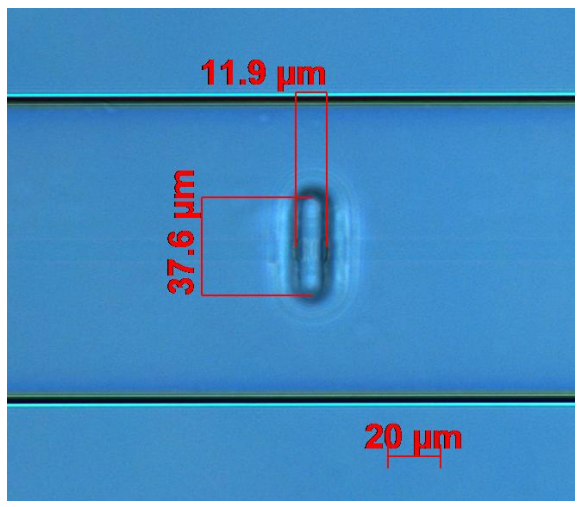

(b)

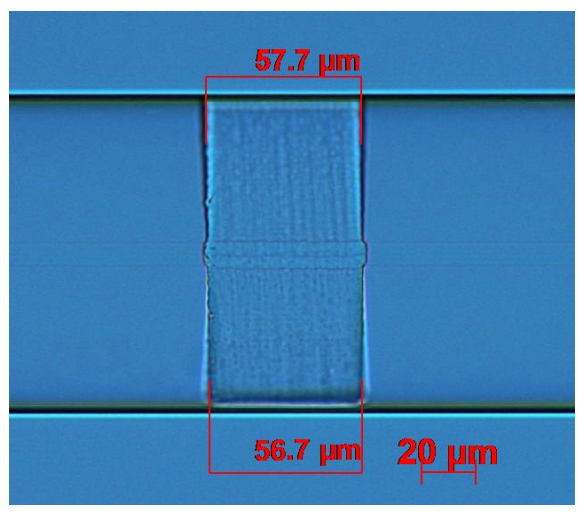

(d)

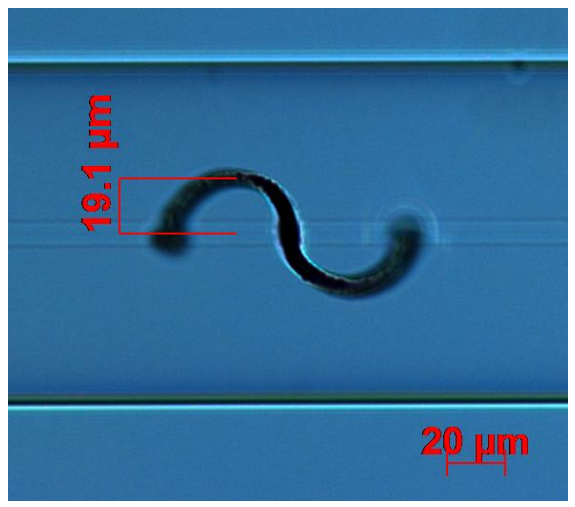

Figure 3. Inscribed and etched microchannel devices - (a) microhole, (b) microslot channel along the core (c) microslot channel perpendicular to the core and (d) single spiral channel around the core.

The R.I. sensitivity of each microchannel device was evaluated through immersing each microchannel in a range of index matching oils (Cargille Labs) and measuring the optical transmission losses. A total of twelve index matching oils were tested in each channel -1.300 to 1.456 . For R.I. oils from 1.30 to 1.38 , the single spiral microchannel was the least sensitive device with a sensitivity of $0.51 \mathrm{~dB} / \mathrm{RIU}$, due to the microchannel being confined only to the cladding. Whereas the microslot channel perpendicular to the core was the most sensitive at $1.55 \mathrm{~dB} / \mathrm{RIU}$. It should be noted that a $0.14 \mathrm{~dB}$ transmission loss was observed through the microslot channel perpendicular to the core when the channel was filled with a 1.30 R.I. oil, whereas the transmission loss was negligible through the single spiral microchannel filled with the same index oil.

Furthermore, an $8 \mu \mathrm{m}$ diameter microhole channel was combined with two $2 \mathrm{~mm} 2^{\text {nd }}$ order FBGs separated by $1 \mathrm{~mm}$ to form an alternative measurement configuration. Both the FBGs and the microslot were inscribed in a single pass. For this particular device, the FP refractometer had a R.I. sensitivity of $2.75 \mathrm{~nm} / \mathrm{RIU}$ and measures a wavelength shift as opposed to a transmission loss. A section of the point-by-point inscribed FBG and the spectral response of the FP refractometer is shown in Figure 4. 
(a)

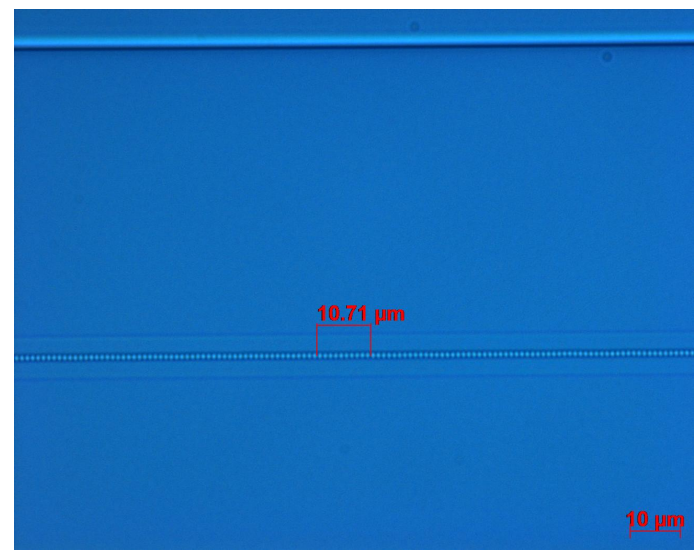

(b)

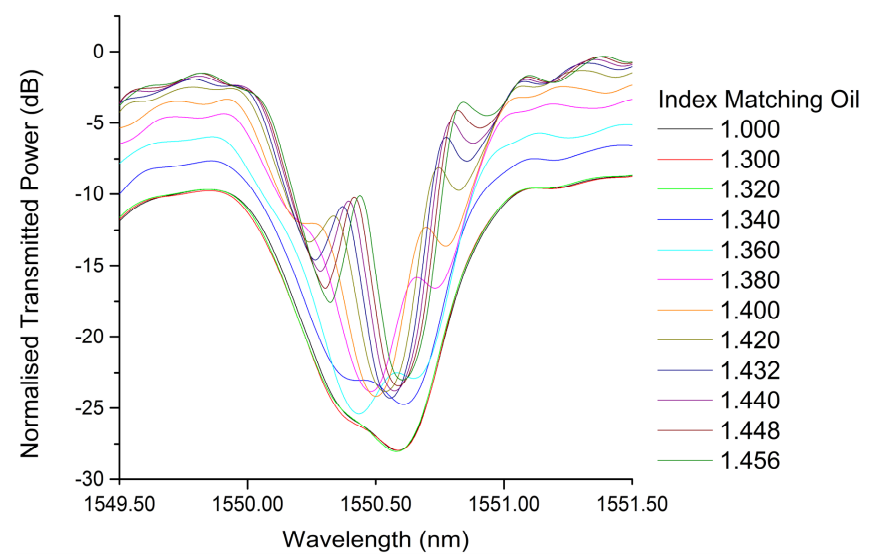

Figure 4. (a) Optical microscope image showing a section of the point-by-point $2^{\text {nd }}$ order $1550 \mathrm{~nm}$ grating. (b) Fabry-Pérot based refractometer - averaged and normalized optical transmission losses measured with index matching oils in the microhole. Measured using a LUNA Optical Vector Analyzer with a 1.25 pm wavelength resolution.

\section{CONCLUSION}

Femtosecond laser inscribed and hydrofluoric etched microchannel designs for liquid sensing has been demonstrated. The fabrication parameters have been optimized to produce high quality devices and a more complex design space has been explored to enable the fabrication of $3 \mathrm{D}$ devices. A R.I. sensitivity up to $1.55 \mathrm{~dB} / \mathrm{RIU}$ was achieved using the microslot channel perpendicular to the core. A simple FP based refractometer with a microslot channel perpendicular to the core yielded an R.I. sensitivity of $2.75 \mathrm{~nm} /$ RIU. Further investigations are underway to incorporate and test the other three microchannel types in the FP refractometer design. The FP device was made using a single laser platform allowing the FBGs and slot to be accurately positioned relative to each other. In addition, further tests are required to determine the suitability of the microchannel devices for gas sensing.

\section{REFERENCES}

[1] Jensen, J. B., Pedersen, L. H., Hoiby, P. E., Nielsen, L. B., Hansen, T. P., Folkenberg, J. R., Riishede, J., Noordegraaf, D., Nielsen, K., Carlsen, A., and Bjarklev, A., "Photonic crystal fiber based evanescent-wave sensor for detection ofbiomolecules in aqueous solutions," Opt. Lett., 29(17), 1974-1976 (2004).

[2] Lien, V., and Vollmer, F., "Microfluidic flow rate detection based on integrated optical fiber cantilever," Lab on a Chip, 7(10), 1352-1356 (2007).

[3] Perrotton, C., Westerwaal, R. J., Javahiraly, N., Slaman, M., Schreuders, H., Dam, B., and Meyrueis, P., “A reliable, sensitive and fast optical fiber hydrogen sensor based on surface plasmon resonance," Opt. Express, 21(1), 382-390 (2013).

[4] Davis, K. M., Miura, K., Sugimoto, N., and Hirao, K., "Writing waveguides in glass with a femtosecond laser," Opt. Lett., 21(21), 1729-1731 (1996).

[5] Lai, Y., Zhou, K., Zhang, L., and Bennion, I., "Microchannels in conventional single-mode fibers," Opt. Lett., 31(17), 2559-2561 (2006).

[6] Martinez, A., Dubov, M., Khrushchev, I., and Bennion, I., "Direct writing of fibre Bragg gratings by femtosecond laser," Electronics Letters, 40(19), 1170-1172 (2004). 